\title{
Sense-Bandits: AI-based Adaptation of Sensing Thresholds for Heterogeneous-technology Coexistence Over Unlicensed Bands
}

\author{
Mohammed Hirzallah and Marwan Krunz \\ ${ }^{1}$ Department of Electrical and Computer Engineering, University of Arizona, AZ, USA \\ Email: \{hirzallah, krunz\}@email.arizona.edu
}

\begin{abstract}
In this paper, we present Sense-Bandits, an AI-based framework for distributed adaptation of the sensing thresholds (STs) over shared spectrum. This framework specifically targets the coexistence of heterogenous technologies, e.g., Wi-Fi, 4G Licensed-Assisted Access (LAA), and 5G New Radio Unlicensed (NR-U), over unlicensed channels. To access the channel, a device compares the measured power with a predefined ST value and accordingly decides if the channel is idle or not. Improper setting of the ST values creates asymmetric sensing floors, resulting in collisions due to hidden terminals and/or reduction in the spatial reuse due to exposed terminals. Optimal ST setting is challenging because it requires global knowledge of mobility, traffic loads, and channel access behavior of all contending devices. SenseBandits tackles this problem by employing a clustering-based multi-armed bandit (MAB) algorithm, which adapts its learning behavior based on network dynamics. Clustering allows the algorithm to track network changes in real-time, ensuring fast learning of the best $S T$ values by classifying the state and dynamics of coexisting networks. We develop a C++-based network simulator that implements Sense-Bandits and we apply it to evaluate the coexistence of Wi-Fi and $5 G$ NR-U systems over the unlicensed $5 \mathrm{GHz}$ U-NII bands. Our simulation results indicate that ST-adaptive devices employing Sense-Bandits do not harm neighboring devices that adopt a fixed ST value.
\end{abstract}

\section{INTRODUCTION}

5G and beyond cellular networks will host new applications with very demanding requirements in terms of extremely high throughput as well as ultra-reliable and lowlatency transmissions [1]. Licensed spectrum below $7 \mathrm{GHz}$ is overly subscribed and cannot meet the demands of these new applications. Additional spectrum is needed. Although the recently opened millimeter-wave (mmWave) bands offer an abundance of spectrum, transmissions over these bands are highly susceptible to blockage and require complex beamforming/tracking procedures to maintain directional line-ofsight communications. Unlicensed bands below $7 \mathrm{GHz}$, on the other hand, offer about $2 \mathrm{GHz}$ of spectrum. They are partially used by IEEE 802.11-based systems (e.g., Wi-Fi). Mobile network operators (MNOs) are pushing for making these bands available for cellular operation under $4 \mathrm{G}$ Licensed Assisted Access (LAA) and, more recently, 5G New Radio Unlicensed (NR-U) [2][3]. Both technologies would use the unlicensed sub-7 GHz spectrum to supplement their licensed services. NR-U can also be leveraged in a standalone mode, offering great opportunity to new MNOs who do not have their own licensed spectrum [4], [5].
Unlicensed-band cellular operation, including LTE-LAA and 5G NR-U, comes with its own challenges. Chief among them is fair coexistence with other incumbents, particularly Wi-Fi systems. Fair access to the shared channel is strongly dependent on the setting of the sensing threshold (ST), which also affects spatial reuse and channel utilization. To access an unlicensed channel, a device, e.g., Wi-Fi access point (AP), Wi-Fi station (STA), 5G NR-U user equipment (UE), etc., must first execute a Listen-Before-Talk (LBT) procedure, which is essentially a variant of the CSMA/CA protocol. According to LBT, the device senses the channel and compares the received power with a predetermined ST value to determine if the channel is idle. NR-U/LAA and IEEE 802.11based technologies adopt different ST settings that result in heterogeneity of their sensing floors. This creates hidden and exposed nodes, which reduce the total network throughput [6]. For example, IEEE 802.11n/ac-based Wi-Fi devices are set with an energy-based ST value of $-62 \mathrm{dBm}$ to detect unknown signals and a signal-based ST value of $-82 \mathrm{dBm}$ to detect Wi-Fi signals. The IEEE 802.11ax-based devices are set with an additional signal-based ST value, which is variable, to detect Wi-Fi signals coming from overlapping Basic Service Sets (OBSS). LAA and NR-U, on the other hand, adopt a fixed maximum energy-based ST value of $-72 \mathrm{dBm}$. Optimal setting of ST values requires real-time global knowledge about the wireless environment as well as dynamics of neighboring networks, including their mobility and traffic loads.

To maximize network performance, we propose a distributed framework that allows every device to learn its optimal ST value in an online manner with the least possible communication overhead. Employing AI and machine learning (ML) can significantly harmonize coexistence between homogeneous and heterogeneous wireless systems [7], [8], [9], [10]. Our framework, called Sense-Bandits, is based on a multi-armed bandit (MAB) algorithm. MAB algorithms are a class of reinforcement learning (RL) that aim at establishing a balance between exploitation (i.e., maintaining the current ST value) and exploration (i.e., trying new ST values) by minimizing the accumulated regret between actual and optimal rewards. However, traditional MAB algorithms suffer from a long convergence time and inability to cope with fastvarying environments, a.k.a., cold start. For example, when the environment experiences fast changes, the learning outcome 
of the MAB algorithm becomes outdated and misleading to the adaptation process. To cope with this challenge, SenseBandits allows devices to detect changes in the environment dynamics and re-initiate the learning process properly by setting the initial values of different arms (actions). This reinitialization utilizes the history of previous experience and copes with the rate at which the environment changes. To make the adaptation process aware of real-time changes, each device constructs over time a feature representation called of the environment a sensing fingerprint $(S F)$. The SFs are used to track the environment and detect real-time changes by finding the similarity between SFs collected over consecutive time periods. Once a change in the environment is detected, the device follows a rule to decide whether the most recent learning outcomes are outdated and whether the device needs to re-initiate its learning process. To reduce the overhead of re-initializing the learning process, we let the device exploit its previous/stored history of learning experience. This is achieved by finding states that are already learned and have close similarity with the new state of the environment. SenseBandits also considers the important tradeoff between utilizing historical learning experiences and limiting the storage and computational requirements at a device. Specifically, the environment may give rise to a huge number of states, making the learning experience of these states computationally prohibitive. By completely ignoring the learning experience, we run into the shortcomings of traditional MAB algorithms. On the other hand, maintaining the complete history of the learning experience incurs high memory and computational overheads. In Sense-Bandits, we focus on finding a balance between these two extremes by clustering the history of learning instances into a finite number of clusters based on the similarity of their underlying states. Our clustering-based multi-armed bandit (CMAB) algorithm accommodates this balance by finding the nearest cluster that has a similar state to the new environment. We associate with every cluster a recommended initial values of STs. Compared with traditional MAB algorithms, CMAB has a faster convergence and can cope better with various network dynamics.

The rest of the paper is organized as follows. In Section III we review related works on ST adaptation. The system model and problem formulation are discussed in Section III Sense-Bandits is presented in Section IV Evaluation of SenseBandits in a simulated Wi-Fi / 5G NR-U coexistence scenario is presented in Section $\mathrm{V}$, followed by conclusions in Section VI. Throughout the paper, we use the superscript $(\cdot)^{(u)}$ to refer to NR-U and $(\cdot)^{(w)}$ to refer to $\mathrm{Wi}-\mathrm{Fi}$.

\section{RELATED WORK}

Adapting STs for harmonious coexistence between LAA and $\mathrm{Wi}-\mathrm{Fi}$ systems has been previously investigated. Li et al. [11] investigated adapting the ST value for LAA devices by increasing or reducing it based on the collision rate. Iqbal et al. [12] investigated the impact of changing the ST values in LAA and Wi-Fi devices, and concluded that lowering these values for Wi-Fi could improve the throughput of both networks.
Ajami et al. [13] analyzed the coexistence between LTE and Wi-Fi systems using stochastic geometry, and suggested that LTE could be a good neighbor to Wi-Fi if the later adapts its ST. Mehrnoush et al. [14] modeled the impact of changing the ST values using Markov-models. While these works offer great insights into ST adaptation, the approaches are either ad hoc or focus mainly on evaluating the effect of changing the ST value rather than adapting it online.

Adapting ST values to improve the spatial frequency reuse has also been discussed in the context of IEEE 802.11 networks. Most of the works assume homogeneous devices (e.g., $\mathrm{Wi}-\mathrm{Fi}$ ) and require these devices to be able to decode certain fields, e.g., Basic Service Set (BSS) color bit. Kulkarni et al. [15] presented extensive evaluation to show the impact of adapting the ST values on improving IEEE 802.11ac network throughput. In [16], the authors investigated improving IEEE 802.11ax network performance by using dual ST values, one is conservative and targets the detection of intra-BSS signals while the other is aggressive and targets the detection of interBSS signals. The authors in [17] demonstrated that adapting the ST values is needed and the best ST value is scenariodependent. Afaqui et al. [18] proposed a framework to adapt the ST values in IEEE 802.11ax networks based on the received interference generated by nearby APs. Selinis et al. [19] presented a framework, called Damysus, for adapting ST values based on the color bit. Recently, researchers started investigating the use of learning techniques to adapt the ST value in IEEE 802.11ax networks. The authors in [20] presented a MAB-based framework to the control ST value, transmit power, and channel selection so as to improve the spatial reuse over IEEE 802.11ax networks. They also demonstrated the advantage of MAB in achieving collaboration among APs to efficiently adapt their ST values [21]. Although these works present exciting results, they are still focused on a homogeneous technology whereby devices can decode each other's frames and read, for example, the color bit to distinguish between signals coming from different BSSs. These assumptions are not applicable to heterogeneous coexistence setting, e.g., NR-U and Wi-Fi coexistence. In contrast, our Sense-Bandits approach is technology-agnostic and can be applied to all technologies sharing an unlicensed channel.

\section{System Model And Problem Formulation}

Without loss of generality, we consider two coexisting networks: A $5 \mathrm{G}$ NR-U network that consists of a set $\mathcal{B}=$ $\left\{B_{1}, \cdots, B_{N_{b}}\right\}$ of $N_{b}$ base stations (BSs) and serves a set $\mathcal{U}=\left\{U_{1}, \cdots, U_{N_{u}}\right\}$ of $N_{u}$ UEs, and a Wi-Fi network that consists of a set $\mathcal{P}=\left\{P_{1}, \cdots, P_{N_{p}}\right\}$ of $N_{p}$ APs and serves a set $\mathcal{S}=\left\{S_{1}, \cdots, S_{N_{s}}\right\}$ of $N_{s}$ STAs. Let $\mathcal{N}=\mathcal{U} \cup \mathcal{B}$ and let $\mathcal{W}=\mathcal{S} \cup \mathcal{P}$. UEs (STAs) attach to the BS (AP) that provides the strongest signal. Our formulations and evaluations are based on single-antenna devices, but can be easily extended for MIMO operation. 5G NR-U and Wi-Fi network share an arbitrary unlicensed channel of bandwidth $W_{c}$ in Hz. An LBT procedure (CSMA/CA with exponential backoff) is used for channel access at a timing granularity of $\Delta_{c}$, where $\Delta_{c}=9$ 
microseconds corresponds to the duration of a MAC time slot. This is inline with NR-U specifications and IEEE 802.11 standards for operating over the $5 \mathrm{GHz}$ UNII bands [22][23].

To ensure the channel is idle, a device compares the sensed signal power with a predefined ST value, a.k.a., detection threshold or clear channel assessment (CCA) threshold. If the channel remains idle for a period of time, a.k.a., Arbitration Inter-Frame Space (AIFS) (or Initial Deferment period), the device starts transmission; otherwise, it initiates a counter with a random value $k$ and backs off for $k$ idle time slots, where:

$$
k \sim \text { uniform }\left\{0, \cdots, \min \left(2^{\varsigma} W_{\min }, W_{\max }\right)-1\right\}
$$

$W_{\min }$ and $W_{\max }$ are the minimum (CWmin) and maximum (CWmax) size of the contention window, respectively, and $\varsigma$ is the retransmission attempt. Let $W_{\min }^{(u)}, W_{\max }^{(u)}$, and $a^{(u)}$ be the CWmin, CWmax, and AIFS values adopted in the NR$\mathrm{U}$ network, respectively. $W_{\min }^{(w)}, W_{\max }^{(w)}$, and $a^{(w)}$ are defined similarly for the Wi-Fi network. During the backoff time, if the channel becomes busy, the device must freeze its counter and await for the channel to become idle again. The device can only decrease its counter if the channel is deemed to be idle. This decision clearly depends on the ST value. Let $\gamma_{j}^{(u)}$ be the ST value adopted by NR-U device $j \in \mathcal{N}$ and $\gamma_{l}^{(w)}$ be the $\mathrm{ST}$ value adopted by $\mathrm{Wi}-\mathrm{Fi}$ device $l \in \mathcal{W}$. In our work, we consider a range of $\gamma_{j}^{(u)}$ and $\gamma_{l}^{(w)}$ values that covers the ST values adopted in the standards, including both energy and signal detection thresholds. The sensed signal power $y_{i}^{(u)}(n)$ at an arbitrary time slot $n$ by an arbitrary UE $U_{i}$ is given by (similar expressions can be formulated for APs, STAs and BSs):

$$
\begin{aligned}
y_{i}^{(u)}(n) & =\sum_{\substack{j \in \mathcal{N} \\
j \neq i}} \Upsilon_{j}^{(u)}(n) h_{j i}(n)\left|s_{j}^{(u)}(n)\right|^{2} \mathbf{1}_{j}^{(u)}(n) \\
& +\sum_{l \in \mathcal{W}} \Upsilon_{l}^{(w)}(n) h_{l i}(n)\left|s_{l}^{(w)}(n)\right|^{2} \mathbf{1}_{l}^{(w)}(n)+z_{i}^{(u)}(n)
\end{aligned}
$$

where $\Upsilon_{l}^{(w)}(n)$ and $\Upsilon_{j}^{(u)}(n)$ are the transmit power of an arbitrary Wi-Fi device $l$ and NR-U device $j$ at time $n, s_{j}^{(w)}(n)$ and $s_{l}^{(u)}(n)$ are the transmit signals of an arbitrary Wi-Fi device $l$ and NR-U device $j$ at time $n, h_{j i}(n)$ is the channel gain between transmitting device $j$ and receiving device $i$ at time $n$, respectively, and $z_{i}^{(u)}(n)$ is the additive white Guassian noise at NR-U device $i . \mathbf{1}_{j}^{(u)}(n)$ and $\mathbf{1}_{l}^{(w)}(n)$ are the indicator functions that NR-U device $j$ and Wi-Fi device $l$ access the unlicensed channel at an arbitrary time $n$, respectively:

$$
\begin{array}{r}
\mathbf{1}_{j}^{(u)}(n)=\left\{1: \text { if } y_{j}^{(u)}(n) \leq \gamma_{j}^{(u)} \text { and } k_{j}^{(u)}(n)=0\right\} \\
\mathbf{1}_{l}^{(w)}(n)=\left\{1: \text { if } y_{l}^{(w)}(n) \leq \gamma_{l}^{(w)} \text { and } k_{l}^{(w)}(n)=0\right\}
\end{array}
$$

where $k_{j}^{(u)}(n)$ and $k_{l}^{(w)}(n)$ are the backoff counter for NRU device $i$ and Wi-Fi device $l$ at time slot $n$, and they are initialized as in (1). There are several factors that affect the setting of these indicator functions, including the ST value used by neighboring devices as well as their channel access behavior, traffic loads, and mobility patterns [24]. Expressing these indicator functions based on these factors requires notoriously complicated stochastic-geometry analysis [13].

\section{A. Problem Formulation}

At time $n$, the uplink throughput $S_{j}^{(u)}(n)$ for an arbitrary UE $U_{j}$ attached to an arbitrary BS $B_{i}$ can be expressed as (similar expressions can be formulated for NR-U downlink as well as Wi-Fi uplink and downlink communications):

$$
S_{j}^{(u)}(n)=W_{c} \mathbb{E}\left[\log \left(1+\frac{Y_{i}(n)}{I_{i}(n)+z_{i}^{(u)}(n)}\right)\right]
$$

where $Y_{i}(n)$ and $I_{i}(n)$ are the received signal power and interference power received by $\mathrm{BS} B_{i}$, respectively:

$$
\begin{aligned}
Y_{i}(n) & =\Upsilon_{j}^{(u)}(n) h_{j i}(n)\left|s_{j}^{(u)}(n)\right|^{2} \mathbf{1}_{j}^{(u)}(n) \\
I_{i}(n) & =\sum_{\ell \in \mathcal{N}, \ell \neq j} \Upsilon_{\ell}^{(u)}(n) h_{\ell i}(n)\left|s_{\ell}^{(u)}(n)\right|^{2} \mathbf{1}_{\ell}^{(u)}(n) \\
& +\sum_{l \in \mathcal{W}} \Upsilon_{l}^{(w)}(n) h_{l i}(n)\left|s_{l}^{(w)}(n)\right|^{2} \mathbf{1}_{l}^{(w)}(n) .
\end{aligned}
$$

The expectation in (5) accounts for the randomness in the interference generated by neighboring devices due to their mobility, traffic loads, and channel access behavior. Our objective is to maximize the sum-throughput experienced by both NR-U and Wi-Fi devices over a period of $T_{n}$ time slots:

$$
\begin{aligned}
P_{1}: \underset{\Gamma^{(u)}(n), \Gamma^{(w)}(n)}{\arg \max } & \sum_{n=1}^{T_{n}}\left[\sum_{j \in \mathcal{N}} S_{j}^{(u)}(n)+\sum_{l \in \mathcal{W}} S_{l}^{(w)}(n)\right] \\
\text { s.t. } \quad & \gamma_{j}^{(u)}(n), \gamma_{l}^{(w)}(n) \in \Gamma, l \in \mathcal{W}, j \in \mathcal{N}
\end{aligned}
$$

where $S_{l}^{(w)}(n)$ is the throughput achieved by Wi-Fi device $l$ at time $n, \Gamma=\left\{\gamma_{1}, \cdots, \gamma_{N_{a}}\right\}$ is the set of possible ST values that can be selected by NR-U and Wi-Fi devices, $\Gamma^{(u)}(n)=$ $\left\{\gamma_{j}^{(u)}(n) \mid j \in \mathcal{N}\right\}$, and $\Gamma^{(w)}(n)=\left\{\gamma_{l}^{(w)}(n) \mid l \in \mathcal{W}\right\}$. The decision variables are the ST values $\Gamma^{(n)}(n)$ for NR-U devices and $\Gamma^{(w)}(n)$ for Wi-Fi devices. The above optimization is stochastic and nonlinear. In principle, it can be solved via dynamic programming. However, such an approach gives rise to several challenges:

- Expressing the objective function as a function of ST values is mathematically intractable and putting it in a closed form is not possible.

- Solving the dynamic programming problem requires global knowledge about the dynamics of NR-U and WiFi networks, including channel conditions, location information, traffic loads, the state of backoff counters, etc. Obtaining this global knowledge is practically difficult due to the large communication overhead and privacy concerns.

- Problem $P_{1}$ involves taking decisions over time, and solving this problem in a distributed fashion requires coordination and synchronization between devices. Due to the differences in the waveforms and message formats between Wi-Fi and 5G NR-U, achieving this coordination/synchronization is difficult. 
In contrast to an exact dynamic programming approach, reinforcement learning offers heuristics, including MABs, to solving $P_{1}$ by employing a learning agent. Specifically, in a $\mathrm{MAB}$, the learning agent aims at finding a balance between exploiting ST values of known average reward and exploring new ones. The learning process focuses on minimizing the accumulated regret expressed by the difference between the average rewards (e.g., throughput) of the optimal and actual ST values. Most well-known MAB algorithms, e.g., upper confidence interval (UCB), epsilon greedy, Thompson sampling, etc., are subject to long learning and convergence times. In a time-varying environment, such as a dynamic unlicensed channel, these algorithms fall short of approaching the optimal solution. Sense-Bandits overcomes this limitation and ensures fast adaptation by employing clustering of the environment and running a cluster-based MAB (CMAB) algorithm. This CMAB algorithm gives the learning agent awareness of the channel and network dynamics, and provides it with prior information required to speed up the learning process.

\section{Sense-BAndits Design}

In Sense-Bandits, an LBT device (e.g., NR-U or Wi-Fi device) runs its own learning gent and takes actions in a distributed fashion. For ease of illustration, we drop the subscript that denotes the device index and explain the formulation from a single device perspective. Our formulation applies to NR-U and Wi-Fi devices. In our subsequent notation, we let the first subscript index denote the time and the second subscript index denote the state of the environment or action, as applicable.

\section{A. Learning Model}

1) Time Horizon: We consider a finite-time horizon $\mathcal{T}=$ $\{1, \cdots, T\}$ that consists of $T$ time epoch. We also use $t \in \mathcal{T}$ to denote the index of time epochs. Every epoch has a fixed duration of $\Delta_{p}$ seconds. Selection of ST value should take place at the start of the time epoch, and during this time epoch the device collects observations and monitors the achieved performance. These observations and performance are used to trigger the selection of ST value to be used over the next time epoch.

2) State of Environment: The state of the environment represents the unlicensed wireless environment, including dynamics of coexisting networks, such as their location information, mobility pattern, traffic loads, channel access behavior, etc. Wi-Fi and NR-U devices should select their best ST values independently based on how they view the state of the wireless environment with the least communication overhead possible. Achieving global knowledge about the state of environment by NR-U and Wi-Fi devices is difficult due to the challenges discussed before. Therefore, instead of obtaining a global knowledge, every device constructs a sensing fingerprint (SF) in which a normalized histogram of the sensed signal powers is constructed over a monitoring period $\Delta_{m}$, where $\Delta_{m} \leq \Delta_{p}$. For example, the monitoring period of the $t$ th epoch can be started in the middle of $t$ th time epoch. In Figure 1, we show an example in which the start of monitoring period aligns with epoch boundary. The monitoring period is divided to sensing periods $\Delta_{s}$ in which the sensed signal power is to be computed, where $\Delta_{s} \ll \Delta_{m}$. One option is to set the sensing period to be equal to MAC time slot $\Delta_{s}$, i.e., $\Delta_{s}=\Delta_{c}=9$ microseconds. The sensing is supposed to take place during the backoff process and to be suspended during the transmission time. Let $e_{t}=\left\langle\tilde{e}_{t, 1}, \cdots, \tilde{e}_{t, N_{r}}\right\rangle$ be the normalized histogram that represents the state of the environment as observed by the device at time epoch $t$, where $N_{r}$ is the number of bins and $\tilde{e}_{t, j} \in[0,1]$ is the probability that the sensed signal power is in the $\left[\delta_{j}, \delta_{j+1}\right)$ interval. Note that $e_{t}$ is a simplex in $\mathbb{R}^{N_{r}}$, i.e., $\sum_{j=1}^{N_{r}} \tilde{e}_{t, j}=1$. The overhead required to construct the state of the environment is practical because both 3GPP specifications and IEEE 802.11 standards require wireless devices to track statistics of their sensed signal powers and report them back to base station and access point. In our work, devices will utilize their sensed signal power locally and they are not supposed to exchange it with their home base station or access point. Characterizing the state of the environment using the SF profile takes advantage of the sensing part that happens during the backoff process, and thus it requires no significant overhead.

3) Actions: We let $\Gamma=\left\{\gamma_{1}, \cdots, \gamma_{N_{a}}\right\}$ be the set of $N_{a}$ possible actions that represent the ST values that a device can use while contending for a channel access. Let $a_{t}$ be the ST value, i.e., action or arm, that the device uses while contending for a channel access during time epoch $t$, where $a_{t} \in \Gamma$. Let $x_{t, a}$ be the action vector at time epoch $t$, where $\left\|x_{t, a}\right\|_{2} \leq d_{x}$, $d_{x}=1$. In our work, actions are selected with hard decision. The size of $x_{t, a}$ is equal to the number of actions, i.e., ST values to be considered as actions. For example, when $\gamma_{j}$ is selected, then the $j$ th element in $x_{t, a}$ will be set to one, while other elements are set to zeros.

4) Rewards: We let the effective throughput achieved during a time epoch be the reward observed by the learning agent. The effective throughput per epoch can be calculated by taking the difference between successful and failed traffic exchanged in one epoch divided by its duration. It is clear that the reward received at a particular time epoch is random and depends on the state of the environment, including ST values, i.e., actions, taken by different devices sharing the same channel. Let $R_{t, e, a}$ be a random variable that represents the reward received by the device, expressed by the effective throughput achieved during time epoch $t$, for taking an action $a$ while the state of the environment is $e$ 1 . For ease of illustration, we drop the time index in the subscript of $e$ and $a$, and time information can be inferred from their associated quantity. Let $r_{t, e, a}^{(s)}$ and $r_{t, e, a}^{(f)}$ be the amount of traffic (expressed in bits) exchanged with success, i.e., with ACK, and with failure, i.e., NACK/ACKtimout, respectively, during time epoch $t$ while taking an action $a$. The sampled reward, $r_{t, e, a} \sim R_{t, e, a}$, as observed by the learning agent can be expressed as:

$$
r_{t, e, a} \leftarrow\left(r_{t, e, a}^{(s)}-r_{t, e, a}^{(f)}\right) / \Delta_{p}
$$

\footnotetext{
${ }^{1}$ For latency stringent applications, the reward can be formulated based on latency experienced during time epoch $t$, and thus the problem in 8 becomes a maximization of the summation of the inverse of delays.
} 
It is a common practice to model the random reward, $R_{t, e, a}$, by a linear function, a.k.a., linear bandits [25]. The reward at time epoch $t$ can be modeled using the following linear relation:

$$
R_{t, e, a} \doteq \mu_{t, e}^{\top} x_{t, a}+\epsilon_{t, e}
$$

where $\mu_{t, e}$ is a vector that represent actions utility for state $e$, i.e., the average rewards of actions when the environment is at state $e$, where $\left\|\mu_{t, e}\right\|_{2} \leq d_{\mu}$. In other words, the $i$ th element in $\mu_{t, e}$ represents the average reward for the $i$ th action. Actions utility $\mu_{t, e}$ is unknown to the learning agent because of the noise $\epsilon_{t, e}$, where noise has a zero mean and $\nu$-sub-Gaussian tail:

$$
\forall \lambda \in \mathbb{R}, \quad \mathbb{E}_{\epsilon_{t, e}}\left[e^{\lambda \epsilon_{t, e}} \mid \mathbf{x}_{1: t}, \mathbf{e}_{1: t}\right] \leq \exp \left(\lambda^{2} \nu^{2} / 2\right)
$$

where $\lambda$ and $\nu$ are the parameters of the $\nu$-Sub Gaussian noise $\epsilon_{t, e}, \mathbf{x}_{1: t}$ is the sequence of previous action history and $\mathbf{e}_{1: t}$ is the sequence of previous noise history. It is obvious that the mean of reward is $\mu_{t, e}^{\top} x_{t, a}$.

\section{B. Selection of Optimal ST Value}

Let $a_{t}^{*}$ be the optimal ST value that the device should select at time epoch $t$ to maximize its expected reward at time $t$. The optimal action $a_{t}^{*}$ can be expressed as follows:

$$
a_{t}^{*}=\underset{a \in \Gamma}{\arg \max } \mathbb{E}\left[R_{t, e, a}\right]=\underset{a \in \Gamma}{\arg \max } \mu_{t, e}^{\top} x_{t, a}
$$

The learning agent does not know the true action utility, i.e., $\mu_{t, e}$, and hence it incurs a regret due to selection of a nonoptimal ST value, say $a \neq a^{*}$. Let $g_{t}$ be the expected regret at time epoch $t$ that can be expressed as:

$$
g_{t}=\mathbb{E}\left[R_{t, e, a^{*}}-R_{t, e, a}\right]=\mu_{t, e}^{\top} x_{t, a^{*}}-\mu_{t, e^{\top}}^{\top} x_{t, a}
$$

We define the accumulated regret $G(T)$ up until time epoch $T$ to be the sum of expected regrets:

$$
G(T)=\sum_{t=1}^{T} g_{t}=\sum_{t=1}^{T} \mu_{t, e}^{\top} x_{t, a^{*}}-\mu_{t, e}^{\top} x_{t, a}
$$

where the expectation is taken over the randomness of environment states and rewards as expressed by the $H_{t}$-conditioned history, where $H_{t}=$ $\left\{e_{1}, x_{1, a}, r_{1, e, a}, \cdots, e_{t-1}, x_{t-1, a}, r_{t, e, a}, e_{t}\right\}$ is the sequence of state-action-reward history observed and taken by the device up until time epoch $t$.

The problem of finding the optimal actions in (13) is equivalent to finding the sequence of actions, i.e., $\left\{a_{1}, a_{2}, \cdots, a_{T}\right\}$ that minimizes the accumulated expected regret over the $T$ epochs:

$$
\begin{array}{ll}
\underset{\left\{a_{1}, \cdots, a_{T}\right\}}{\arg \min } & G(T) \\
& \text { s.t., } a \in \Gamma
\end{array}
$$

\section{Clustering of States}

We consider a time variant wireless environment where the state of the environment follows a time variant distribution $f_{t, e}$, i.e., $e_{t} \sim f_{t, e}$. We assume the learning agent is unaware of this distribution. We let $E=\left\{e_{t} \mid e_{t} \in \mathbb{R}^{N_{r}}, \sum_{j=1}^{N_{r}} \tilde{e}_{t, j}=1\right\}$ be state space of the environment. We partition the states of environment in $E$ to $N_{c}$ subsets, i.e., clusters, based on their similarities. Let $\mathcal{C}=\left\{E_{1}, \cdots, E_{N_{c}}\right\}$ be the set of the $N_{c}$ disjoint clusters, where $E=\cup_{k=1}^{N_{c}} E_{k}$ and $E_{k} \cap E_{l}=\emptyset$. For each cluster, say $E_{k}$, we define a centroid, $\bar{e}_{k}$, radius, $\bar{d}_{k}$, and average actions utility, $\bar{\mu}_{k}$. One possible approach to cluster states is using K-mean algorithm where the distance between states is expressed based on a similarity measure such as Kullback-Leibler (KL) divergence. Let $e_{t}=\left\{\tilde{e}_{t, 1}, \cdots, \tilde{e}_{t, N_{r}}\right\}$ and $e_{t^{\prime}}=\left\{\tilde{e}_{t^{\prime}, 1}, \cdots, \tilde{e}_{t^{\prime}, N_{r}}\right\}$ be two arbitrary states captured over two different time epochs, i.e., $t$ and $t^{\prime}$. The KL divergence between these two states can be expressed as:

$$
V_{K L}\left(e_{t} \| e_{t^{\prime}}\right) \doteq \sum_{l=1}^{N_{r}} \tilde{e}_{t, l} \log \left(\tilde{e}_{t, l} / \tilde{e}_{t^{\prime}, l}\right)
$$

We can also define average actions utility of a cluster based on the utilities of its constituting states. Let $M_{c}=$ $\left\{\bar{\mu}_{1}, \cdots, \bar{\mu}_{N_{c}}\right\}$ be the set of actions utility of $N_{c}$ clusters, where the average actions utility $\bar{\mu}_{k}$ of cluster $E_{k}$ can be expressed as the average sum of $\mu_{e}$ 's of states in $E_{k}$ :

$$
\bar{\mu}_{k}=\sum_{e \in E_{k}} \mu_{e} /\left|E_{k}\right|
$$

Each cluster can be expressed by its centroid, i.e., center, and its maximum radius. Let $C=\left\{\bar{e}_{1}, \cdots, \bar{e}_{N_{k}}\right\}$ be the set of centroids of the $N_{c}$ clusters, where the centroid $\bar{e}_{k}$ of cluster $E_{k}$ can be expressed as the average sum of states in $E_{k}$ :

$$
\bar{e}_{k}=\sum_{j}^{\left|E_{k}\right|} e_{j} /\left|E_{k}\right|, \quad e_{j} \in E_{k}
$$

Let $D=\left\{\bar{d}_{1}, \cdots, \bar{d}_{N_{c}}\right\}$ be the set of radii of $N_{c}$ clusters. The radius of a cluster can be expressed using KL divergence by finding the maximum divergence between states in cluster and its centroid $\bar{e}_{k}$ :

$$
\bar{d}_{k}=\max \left\{V_{K L}\left(e_{j} \| \bar{e}_{k}\right): e_{j} \in E_{k}\right\}
$$

Finding average actions utilities, $M_{c}$, set of centroids, $C$, and set of radii, $D$, of clusters can be done offline after capturing large number of measurements over diverse set of scenarios. These parameters can be loaded to mobile devices, i.e., UEs and STAs, as lookup tables. It is also possible to let base station and AP construct these parameters online and have them shared with mobile devices through signaling. It should be noted that average action utilities of different clusters, $M_{c}$, can be formulated based on previous learning histories acquired by running traditional MAB algorithm over large number of empirical and/or simulated environments. 
1) Detecting Changes in Environment: Devices can detect substantial changes happening in the environment by tracking the best cluster that describes the environment. To track the best cluster over time, a device should continuously monitor the state $e_{t}$ of the environment and find the nearest cluster, $E_{t}^{*}$, as follows:

$$
E_{t}^{*}=\underset{E_{l} \in \mathcal{C}}{\arg \min } V_{K L}\left(e_{t} \| \bar{e}_{l}\right)
$$

This process has a linear complexity in terms of the number of clusters $N_{c}$, and it becomes expensive when the number of clusters becomes large. A better way to detect changes happening in the environment is to let the device run a detection rule in which it checks whether it deviates from its current cluster. This can be achieved by leveraging the radius of the cluster. Device can compute the similarity of its most recent state $e_{t}$ with the centroid of the current cluster. Let $\bar{e}_{t}$ and $\bar{d}_{t}$ be the centroid and radius of the current cluster at time epoch $t$, respectively. Let $\theta_{t}$ be the indicator function that signals a change in the environment at time $t$, then $\theta_{t}$ can be expressed as follows:

$$
\theta_{t}=\left\{1: V_{K L}\left(e_{t-1} \| \bar{e}_{t-1}\right)>\bar{d}_{t-1}\right\}
$$

When $\theta_{t}$ is set, the device can search for the best cluster as expressed in (21). This will ensure that the detection of changes happening in the environment is of low computational complexity.

2) Reward Formulation Based on Clustering: The linear reward formulation expressed in (11) can be reformulated to take advantage of clustering. Let $E_{k}$ be the best cluster at time epoch $t$, i.e., $E_{t}^{*}=E_{k}$. Let $\bar{\mu}_{t}^{*}$ be the average action utility of cluster $E_{t}$ at time epoch $t$, then $\bar{\mu}_{t}^{*}=\bar{\mu}_{k}$. The random reward $R_{t, e, a}$ can be re-expressed as follows:

$$
R_{t, e, a} \doteq \bar{\mu}_{t}^{* \top} x_{t, a}+\Delta \mu_{t, e}^{\top} x_{t, a}+\tilde{\epsilon}_{t, e}
$$

where $\tilde{\epsilon}_{t, e}$ is the reward noise of cluster $E_{t}^{*}$, i.e., $\tilde{\epsilon}_{t, e}=\tilde{\epsilon}_{k}$, and $\tilde{\epsilon}_{k}$ is the noise of cluster $E_{k}$. The noise per cluster $\tilde{\epsilon}_{t, e}$ could have different statistics from $\epsilon_{t, e}$ in (11), and this depends on the clustering process. Rather than learning the action utility $\mu_{t, e}$ in (11), the learning agent is supposed to learn the fractional action utility $\Delta \mu_{t, e}$ because it already knows $\bar{\mu}_{t}^{*}$. In other words, knowledge about $\bar{\mu}_{t}^{*}$ would help in reducing the impact of noise and have more confidence about the true action utility. The noise of cluster can have similar formulation as in (12) but with different $\lambda_{k}$ and $\nu_{k}$ parameters.

\section{Clustering-Multi-Armed Bandits (CMAB) Algorithm}

We next explain the design and flow of CMAB algorithm. Let $e$ be the state for which its actions utility vector needs to be learned, and let $E_{t}^{*}$ be the best cluster that represents the state of the environment. The goal is to find an estimate for $\mu_{t, e}$ based on the initial knowledge $\bar{\mu}_{t}^{*}$. In other words, the learning agent seeks to find an estimate $\tilde{\mu}_{t, e}$ for the fractional actions utility $\Delta \mu_{t, e}$ in (23). This estimation can be facilitated using a procedure of online linear regression, where $\tilde{\mu}_{t, e}$ can be considered as a standard linear least-squares approximation of $\Delta \mu_{t, e}$. The estimate $\tilde{\mu}_{t, e}$ needs to be updated over time based on observations seen by the learning agent. To update the $\tilde{\mu}_{t, e}$ over time, we need to consider two entities $X_{t}$ and $b_{t, e}$. The $X_{t, e}$ is a matrix used to keep track of the usage of different actions over time, and it can be formulated as:

$$
X_{t, e}=X_{t-1, e}+x_{t-1, a} x_{t-1, a}^{\top}
$$

where $X_{t, e}$ can be initiated to an identity matrix. The $b_{t, e}$ is a vector that tracks the accumulated rewards observed over time, and it can be updated based on the reward observed over previous epoch $r_{t-1, e, a}$ in (10) as follows:

$$
b_{t, e}=b_{t-1, e}+r_{t-1, e, a} x_{t-1, a}
$$

and the initial value of $b_{t, e}$ can be set to a zero vector. Based on $X_{t, e}$ and $b_{t, e}, \tilde{\mu}_{t, e}$ can be expressed as follows:

$$
\tilde{\mu}_{t, e}=X_{t, e}^{-1} b_{t, e}
$$

To consider an upper confidence bound on the estimated utility of an action at time $t$, we consider the following confidence bound $\mathrm{CB}_{t, e}$ [26]:

$$
\mathrm{CB}_{t, e}\left(x_{t, a}\right)=\alpha \sqrt{x_{t, a}^{\top} X_{t, a}^{-1} x_{t, a} \log (t+1)}
$$

where $\alpha$ is the learning parameter that controls the exploration for new actions. It should be noted that the $x_{t, a}^{\top} X_{t, a}^{-1} x_{t, e}$ term in (27) accounts for the inverse of the number of times for which an action has been selected up until time $t$. In other words, the more the action is selected, the more confident is the estimate of its utility, and thus the lower the inflation of its utility, i.e., $\mathrm{CB}_{t, e}$. To select the best action at time epoch $t$, the learning agent should find the action that maximizes the following:

$$
a_{t}=\underset{a \in \Gamma}{\arg \max } \quad \bar{\mu}_{t}^{\top} x_{t, a}+\tilde{\mu}_{t, e}^{\top} x_{t, a}+\mathrm{CB}_{t, e}\left(x_{t, a}\right)
$$

A pseudo-code for the algorithm is given in Algorithm 11 The algorithm starts by determining the best cluster that represents the environment. To do so, the device monitors the unlicensed channel for $\Delta_{m}$ period and constructs an initial $\mathrm{SF} e_{0}$ that represents the initial state of the environment. The device finds the best cluster according to 21], and initializes the centroid $\bar{e}_{0}$, radius $\bar{d}_{0}$, and average actions utility $\bar{\mu}_{0}$, accordingly. The regression quantities $b_{0, e}$ and $X_{0, e}$ are initialized to zero vector and identity matrix. Fractional utility estimate $\tilde{\mu}_{0, e}$ is initialized to zero and the observed reward $r_{0, e, a}$ to zero.

At the start of each time epoch, say epoch $t$, the device performs the following. First, it checks whether changes in environment require switching to a new cluster by running the detection rule in (22). If $\theta_{t}$ is set, device updates centroid, radius, and average utility to match these of the new cluster and re-initializes $X_{t, e}=I$ and $b_{t, e}=\mathbf{0}$. If no substantial change is detected, the device maintains cluster parameters used over the previous epoch. Second, the device updates $X_{t, e}$, $b_{t, e}$, and $\tilde{\mu}_{t, e}$ according to (24), 25), and (26), respectively. Third, the device finds the best action to be used over epoch 


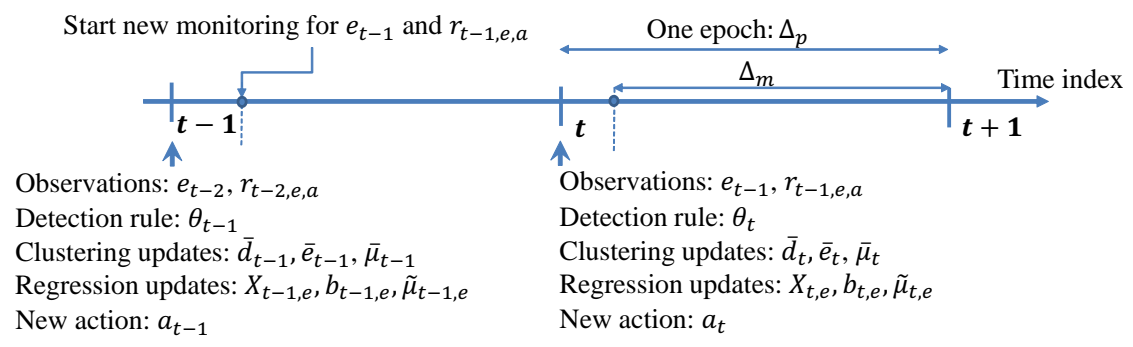

Fig. 1. Timing of different updates over a sequence of time epochs.

$t$ by running the optimization in (28). Once the new action is selected, the device operates normally while monitoring the environment to obtain a new state representation, i.e., $e_{t}$, and reward $r_{t, e, a}$. The timing of different updates is illustrated in Figure 1 .

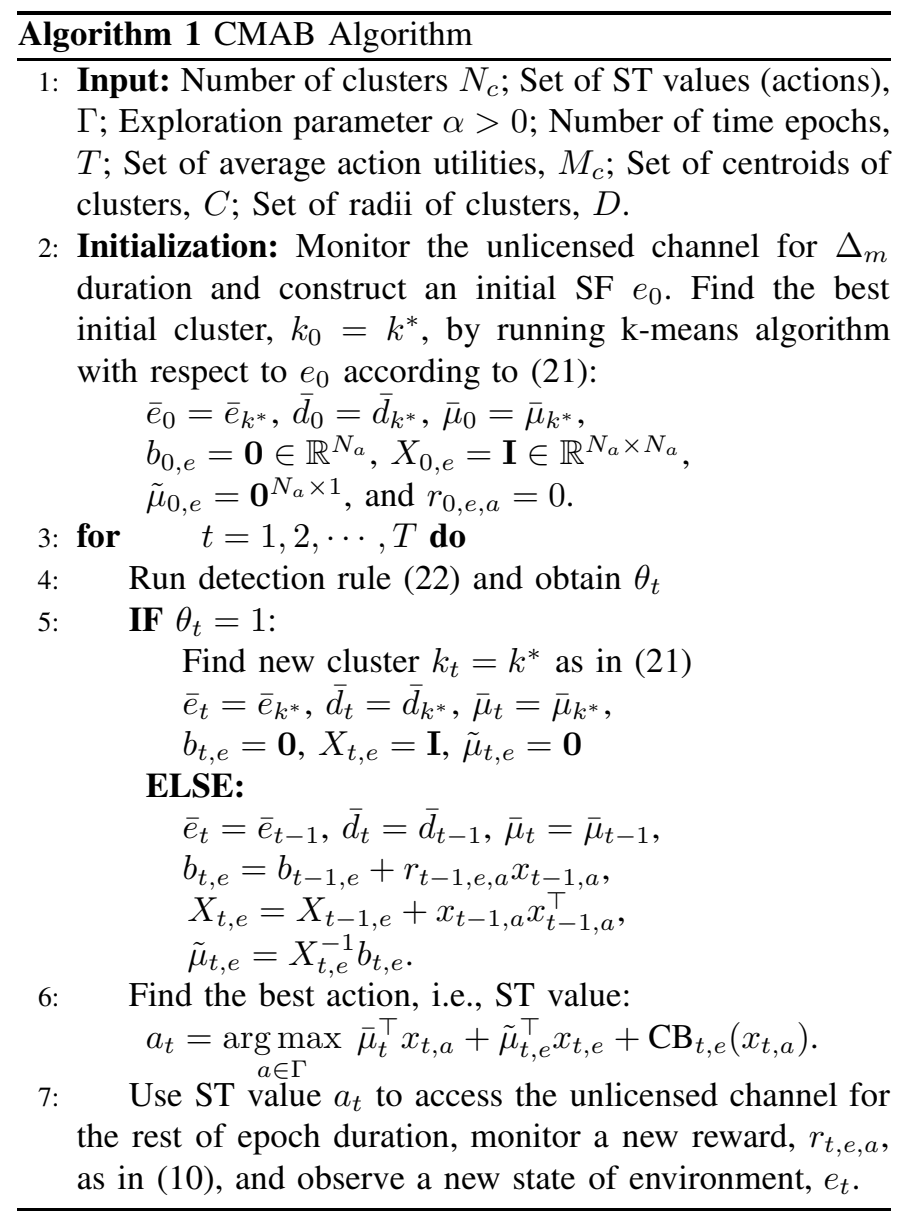

\section{Performance Evaluation and Discussion}

\section{A. Simulation Setup}

We develop a C++-based system-level simulator to study NR-U and Wi-Fi coexistence. Our simulator has been set with an accurate sub-nanosecond timing resolution. The simulator relies on a C++-library, called CSIM, that supports tracking of time, setting events, creating parallel processes, i.e., threads, as well as enabling communications between processes. For each device, we trigger parallel processes for handling various functions, including traffic generation, resource scheduling, LBT-based channel access, transmission over the air, etc. We implement the MAC layer for both NR-U and Wi-Fi networks and model the PHY layer as recommended by the 3GPP technical report [3] and IEEE 802.11 standard [23]. NR-U and Wi-Fi networks share a common channel of $20 \mathrm{MHz}$ bandwidth that is centered at $5.18 \mathrm{GHz}$. We consider the $3 \mathrm{GPP}$ indoor coexistence scenario, as shown in Figure 2 NR-U and Wi-Fi networks have 3 cells, and each cell serves 5 users. $\mathrm{NR}-\mathrm{U}$ and Wi-Fi users are dropped in the simulation area uniformly while ensuring their minimum received power from their home cell is above $-82 \mathrm{dBm}$. The channel is modeled according to 3GPP InH office channel model.

Wi-Fi devices access the channel using the EDCA scheme [23], and NR-U devices use the CAT4-LBT channel access scheme to access the shared channel [22]. Unless stated otherwise, both networks serve FTP traffic with file size 0.5 MB and Poisson arrival. We also analyze various traffic intensity for file arrivals. We also consider a random-walk mobility model for NR-U and Wi-Fi users with maximum speed of 1.5 meters/sec. Unless stated otherwise, Wi-Fi devices access the channel according to the 'Best Effort' access category (AC) with $W_{\min }^{(w)}=16, W_{\max }^{(w)}=1024$, and $a^{(w)}=3$, while NR-U devices, on the other hand, access the channel according to the 3GPP 'Priority Class 2' with $W_{\min }^{(u)}=16, W_{\max }^{(u)}=64$, and $a^{(u)}=3$. The rest of simulation parameters, including shadowing, fading, traffic profile, etc., are set in line with settings in Annex A of 3GPP docs [2][3].

To evaluate the gains of our framework, we compare SenseBandits framework with other two frameworks: Standard and Random framework. In the Standard framework, Wi-Fi and NR-U devices set their ST values according to their standard setting, i.e., $\gamma_{j}^{(w)}=-62 \mathrm{dBm}$ and $\gamma_{i}^{(u)}=-72 \mathrm{dBm}$. In the Random framework, we let NR-U and Wi-Fi devices select their ST values uniformly in the range $\{-82,-81, \cdots,-62\}$ $\mathrm{dBm}$.

\section{B. Time Dynamics}

We investigate the dynamics of $\mathrm{CMAB}$ algorithm over time when compared to other schemes, as shown in Figures 3 and 4 . We evaluate the effective throughput achieved by the three schemes, i.e., CMAB, Standard, and Random. The 


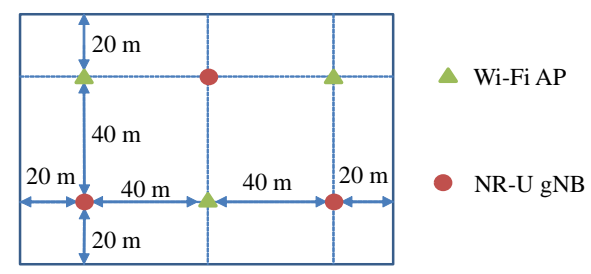

Fig. 2. 3GPP indoor simulation scenario ( $a=20$ meter, $b=40$ meter) 3$]$.

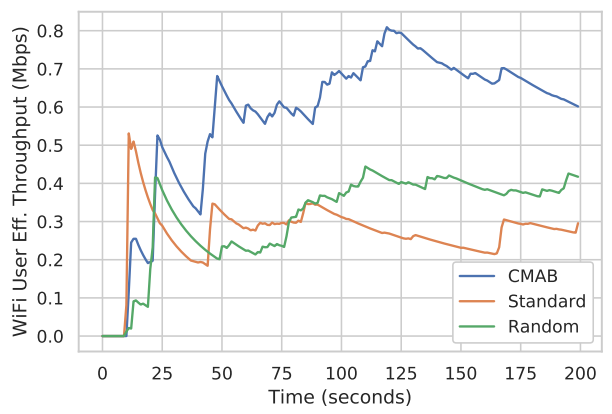

Fig. 3. Wi-Fi user effective throughput vs. time.

effective throughout at a certain moment is computed by dividing the accumulated traffic delivered successfully by the duration of time up until the moment of interest. In Figures 3 and 4, we notice that the CMAB algorithm provides higher throughput than the other two schemes; thanks to the learning feature of CMAB for offering awareness about interference and dynamics of neighboring devices.

\section{Performance Measures}

We evaluate the throughput of CMAB algorithm against the Standard and Random schemes. In our evaluation, we divide users in each cell to two sets, users adapting their ST values, a.k.a., Adapting Nodes, and users fixing their ST values to the standard settings, a.k.a., Standard Nodes. In particular, we let the first three users in every cell adapt their ST values, while the remaining two users fix their ST values to the standard settings. This heterogeneous setup allows us to investigate the impact of adapting ST values under heterogeneous setup

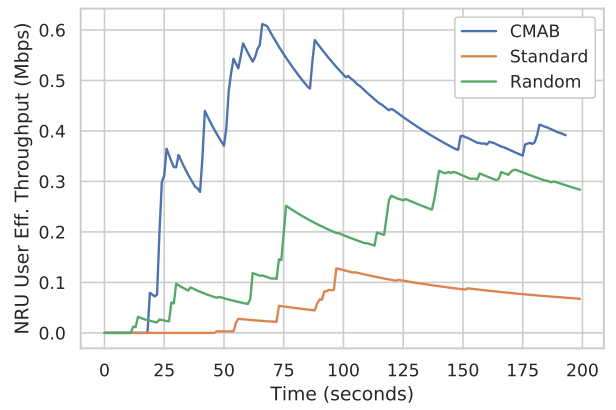

Fig. 4. NR-U user effective throughput vs. time.

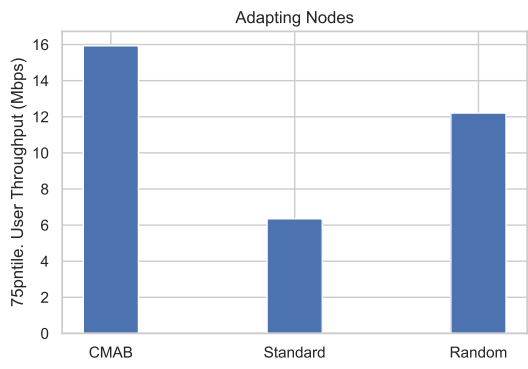

Fig. 5. $75 \%$ percentile of NR-U user perceived throughput for users adapting their ST values.

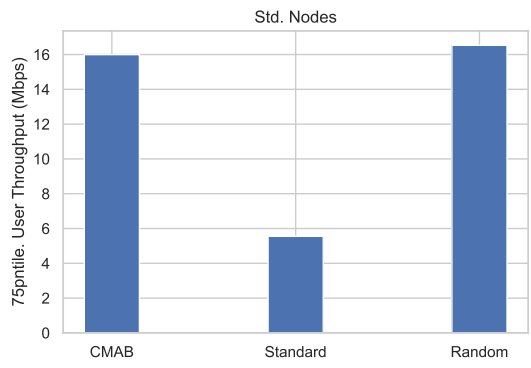

Fig. 6. $75 \%$ percentile of NR-U user perceived throughput for users using fixed standard-compliant ST values.

in which standard devices could exist in the proximity of the intelligent devices. We also report the throughput for both Adapting Nodes and Standard Nodes when the Adapting Nodes run other schemes as well. We investigate the User Perceived Throughput achieved by the three schemes at the MAC layer level. Throughput is calculated by dividing the file size by the time it takes to deliver the file to the receiver. Every file of 0.5 MB is divided into smaller segments (packet) with each segment consisting of $8 \mathrm{~KB}$.

1) NR-U Performance: In Figures [5] and 6, we plot the 75th percentile of throughput for NR-U Adapting Nodes and Standard Nodes, respectively, achieved under the three schemes (i.e., CMAB, Standard, and Random). We observe that CMAB algorithm provides NR-U Adapting Nodes higher throughput than the Standard and Random schemes. We also observe an exciting observation regarding the Random scheme. It can be observed that random assignment of ST values can still provide higher throughput when compared to the standard settings. We also observe that the Standard Nodes experience better throughput when they share the channel with the Adapting Nodes under the CMAB scheme when compared to all-standard setting in which all devices stick to their standard ST values. This proves that adapting ST values improves the performance of both Adapting Nodes and standard-complaint ones as well.

2) Wi-Fi Performance: In Figures [7 and 8, we plot the 75th percentile of Wi-Fi throughput for Adapting Nodes and Standard Nodes, respectively, under the three schemes. We observe the CMAB algorithm provides Adapting Nodes higher 


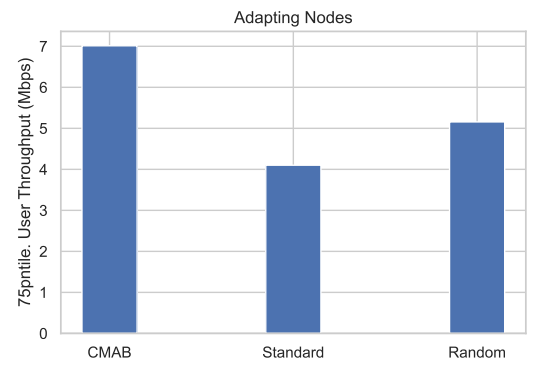

Fig. 7. $75 \%$ percentile of Wi-Fi user perceived throughput for users adapting their ST values.

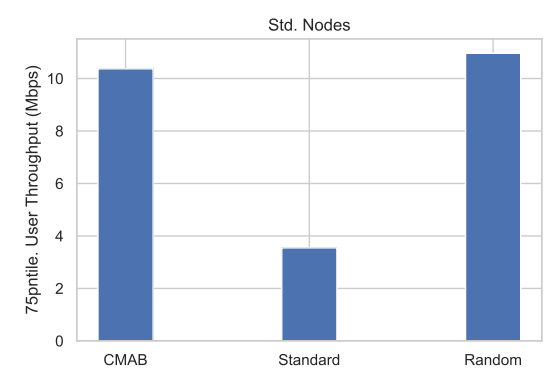

Fig. 8. 75\% percentile of Wi-Fi user perceived throughput for users using fixed standard-compliant ST values.

throughput than the Standard and Random schemes. Once again, it can be observed that the random assignment of ST values still provides higher throughput than the standard settings. The Wi-Fi Standard Nodes experience better throughput when they share the channel with the Adapting Nodes under the CMAB scheme as well.

\section{Conclusions}

In this work, we introduced a new framework for distributed learning and adaptation of ST values used by NR-U and WiFi devices. The new framework relies on a novel clusteringbased multi-armed bandit (CMAB) algorithm. We conducted extensive system-level simulations to evaluate the gains and performance improvement of CMAB as compared to fixed standard as well as random setting of ST values. We spotted many exciting observations. The CMAB algorithm provided higher throughput than the standard settings. In addition, we found that even the random adaptation of ST values could provide higher throughput than fixed settings. The $\mathrm{CMAB}$ algorithm acted as a friendly neighbor to standard-compliant devices that do not adapt their ST values.

\section{REFERENCES}

[1] ITU, "Chairmans report," no. FG IMT-2020, Dec. 2016.

[2] 3GPP, "Study on licensed-assisted access to unlicensed spectrum," 3GPP TR. 36.889 v13.0.0., Jun. 2015.

[3] _ "Study on NR-based access to unlicensed spectrum," no. 3GPP TR 38.889 v16.0.0, Dec. 2018

[4] S. Lagen, L. Giupponi, S. Goyal, N. Patriciello, B. Bojovic, A. Demir, M. Beluri, and J. Mangues-Bafalluy, "New radio beam-based access to unlicensed spectrum: Design challenges and solutions," arXiv preprint arXiv:1809.10443, 2018.
[5] M. Hirzallah, M. Krunz, B. Kecicioglu, and B. Hamzeh, "5G new radio unlicensed: Challenges and evaluation," IEEE Transactions on Cognitive Communications and Networking, pp. 1-1, 2020.

[6] L. B. Jiang and S. C. Liew, "Improving throughput and fairness by reducing exposed and hidden nodes in 802.11 networks," IEEE Transactions on Mobile Computing, vol. 7, no. 1, pp. 34-49, Jan 2008.

[7] M. Hirzallah, W. Afifi, and M. Krunz, "Full-duplex-based rate/mode adaptation strategies for Wi-Fi/LTE-U coexistence: A POMDP approach," IEEE Journal on Selected Areas in Communications, vol. 35, no. 1, pp. 20-29, Jan 2017.

[8] A. H. Y. Abyaneh, M. Hirzallah, and M. Krunz, "Intelligent-CW: AIbased framework for controlling contention window in WLANs," in Proc. of IEEE International Symposium on Dynamic Spectrum Access Networks (DySPAN), 2019, pp. 1-10.

[9] M. Hirzallah, Y. Xiao, and M. Krunz, "Matchmaker: An inter-operator network sharing framework in unlicensed bands," in Proc. of IEEE SECON'19 Conference, June 2019, pp. 1-9.

[10] M. Hirzallah and M. Krunz, "Intelligent tracking of network dynamics for cross-technology coexistence over unlicensed bands," in Proc. of International Conference on Computing, Networking and Communications (ICNC), 2020, pp. 698-703.

[11] L. Li, J. P. Seymour, L. J. Cimini, and C. Shen, "Coexistence of Wi-Fi and LAA networks with adaptive energy detection," IEEE Transactions on Vehicular Technology, vol. 66, no. 11, pp. 10384-10 393, Nov 2017.

[12] M. Iqbal, C. Rochman, V. Sathya, and M. Ghosh, "Impact of changing energy detection thresholds on fair coexistence of Wi-Fi and LTE in the unlicensed spectrum," in Proc. of Wireless Telecommunications Symposium (WTS), April 2017, pp. 1-9.

[13] A. Ajami and H. Artail, "On the modeling and analysis of uplink and downlink IEEE 802.11ax Wi-Fi with LTE in unlicensed spectrum," IEEE Transactions on Wireless Communications, vol. 16, no. 9, pp. 57795795, Sep. 2017.

[14] M. Mehrnoush, V. Sathya, S. Roy, and M. Ghosh, "Analytical modeling of Wi-Fi and LTE-LAA coexistence: Throughput and impact of energy detection threshold," IEEE/ACM Transactions on Networking, vol. 26, no. 4, pp. 1990-2003, Aug 2018.

[15] P. Kulkarni and F. Cao, "Dynamic sensitivity control to improve spatial reuse in dense wireless LANs," in Proc. of the ACM MSWiM Conference, 2016, pp. 323-329.

[16] J. Mvulla and E. Park, "Enhanced dual carrier sensing with transmission time control for fair spatial reuse in heterogeneous and dense WLANs," IEEE Access, vol. 6, pp. 22 140-22 155, May 2018.

[17] O. Oteri, F. La Sita, R. Yang, M. Ghosh, and R. Olesen, "Improved spatial reuse for dense 802.11 WLANs," in Proc. of IEEE Globecom Workshops (GC Wkshps), Dec 2015, pp. 1-6.

[18] M. S. Afaqui, E. Garcia-Villegas, E. Lopez-Aguilera, and D. CampsMur, "Dynamic sensitivity control of access points for IEEE 802.11ax," in Proc. of IEEE ICC Conference, May 2016, pp. 1-7.

[19] I. Selinis, K. Katsaros, S. Vahid, and R. Tafazolli, "Damysus: A practical IEEE 802.11 ax BSS color aware rate control algorithm," International Journal of Wireless Information Networks, vol. 26, no. 4, pp. 285-307, 2019.

[20] F. Wilhelmi, S. Barrachina-Muñoz, B. Bellalta, C. Cano, A. Jonsson, and G. Neu, "Potential and pitfalls of multi-armed bandits for decentralized spatial reuse in wlans," Journal of Network and Computer Applications, vol. 127 , pp. 26-42, 2019.

[21] F. Wilhelmi, C. Cano, G. Neu, B. Bellalta, A. Jonsson, and S. Barrachina-Muñoz, "Collaborative spatial reuse in wireless networks via selfish multi-armed bandits," Ad Hoc Networks, vol. 88, pp. 129-141, 2019.

[22] 3GPP, "Physical layer procedures for shared spectrum channel access," 3GPP TS. 37.213 v16.5.0., Mar. 2021.

[23] IEEE, "IEEE-part 11: Wireless LAN MAC and PHY layer specifications," pp. 1-3534, 2016.

[24] M. Hirzallah, M. Krunz, and Y. Xiao, "Harmonious cross-technology coexistence with heterogeneous traffic in unlicensed bands: Analysis and approximations," IEEE Transactions on Cognitive Communications and Networking, vol. 5, no. 3, pp. 690-701, Sep. 2019.

[25] Y. Abbasi-Yadkori, D. Pál, and C. Szepesvári, "Improved algorithms for linear stochastic bandits," in Advances in Neural Information Processing Systems, 2011, pp. 2312-2320.

[26] P. Auer and R. Ortner, "UCB revisited: Improved regret bounds for the stochastic multi-armed bandit problem," Periodica Mathematica Hungarica, vol. 61, no. 1-2, pp. 55-65, 2010. 